\title{
Fungal Airsporal Contamination of Different Hospital Environments in Lagos, Nigeria
}

\author{
*SAMUEL, TO; KAYODE, YA; ODEWUNMI, OO
}

\author{
Mycology Unit, Department of Botany, University of Lagos, Akoka, Lagos State \\ *Corresponding Author Email: tosamuel@unilag.edu.ng; temmitade@yahoo.com; Tel: +2348036410449
}

\begin{abstract}
Hospital infections caused by fungi have been frequently reported in hospitalized patients, with a high morbidity and mortality, making it increasingly important to the awareness of air quality. This study is designed to isolate, identify and investigate the occurrence of airborne fungal spores present in various working environments of some selected hospitals within Lagos State, Nigeria. Air samples at six working area (reception, out-patient, male ward, female ward, maternity ward and operating theatre) of the selected hospitals were obtained on triplicate of Sabouraud dextrose agar plates, every week days for six consecutive weeks. Isolated fungi were subsequently identified using both microscopic and macroscopic characteristics. Seven species of fungi belonging to three genera were recovered during this study period; Aspergillus niger, A. flavus, A. fumigatus, A. species, Candida species, Penicillium citrinum and P. species. The most abundant fungus encountered in this study is A. niger with P. species being the least isolated fungus from all the investigated hospitals. Of the six working areas sampled in the eight hospitals, the reception and out-patient areas appeared to record the highest occurrence of encountered fungi while the examined operating theatre rooms had the least fungal airsporal contaminant. It was gathered from this study that despite the daily cleaning of the investigated hospital working environments with different antiseptic and disinfectants, fungal contaminant was observed throughout the sampled period. We thereby conclude that the hospital environments are continuously monitored in order to identify and eliminate the sources of these fungal spores.
\end{abstract}

DOI: https://dx.doi.org/10.4314/jasem.v25i5.27

Copyright: Copyright (C) 2021 Samuel et al. This is an open access article distributed under the Creative Commons Attribution License (CCL), which permits unrestricted use, distribution, and reproduction in any medium, provided the original work is properly cited.

Dates: Received: 20 March 2021; Revised: 27 April 2021; Accepted: 07 May 2021

Keywords: Fungi, Airsporal, Hospital environment, Disinfectants, Patients, Lagos.

Fungi are ubiquitous in nature and can be transmitted by wind, water, insects, humans and animals, are able to spread through the ambient air and thereby called airborne (Aglawe et al. 2015). Air is a natural habitat of various fungi, mostly in the form of spores, clusters of spores and fragments of mycelium (Malgorzata, 2014). Fungal spores and fragment of mycelium passively float in the air and are spread by air currents (Southworth, 1974). Fungal spores are discharged either passively or through active mechanisms and become airborne (Tainter and Baker, 1996). These spores may either land on suitable substrates and germinate into new organism or remain dormant for hundreds of years, becoming fossilized with time (Carris et al. 2012). Fungal species that may be present in hospitals, unusual opportunistic fungi in environment such as species of Candida and Aspergillus remain major cause of nosocomial infection (Pfaller and Diekema, 2007). The health hazards associated with exposure to these fungal spores include not only immunosuppressed patients, but also healthy individuals who may develop hyperactivity to the fungal allergens; such as asthma and allergic reaction (Cross, 1997). Approximately, $10 \%$ of all patient infections are suspected to be hospital-acquired (Meers et al. 1990). Many of these infections are endogenous in nature, but others can be acquired from exogenous means by healthcare workers, contaminated infusion products, biomaterials and abiotic environmental sources (Bifarin et al. 2013). The presence of fungal airborne spores in indoor air of hospital environment brings about a challenge to the health of individuals in the hospital which can also lead to hospital acquired infection and prolonged stay in the hospital (Saadoun et al. 2008). Hospital infections caused by fungi have been frequently reported in hospitalized patients, with a high morbidity and mortality, making it increasingly important to the awareness of air quality (Mousavi et al. 2016). Disinfectants and antiseptics are chemicals commonly used for a range of topical and hard surface procedures in hospitals and other healthcare settings (McDonnell and Russell, 2001). The British Standards Institution further define disinfection as not necessarily killing all of the organisms, but reducing them to a level which is neither harmful to health nor to the quality of perishable goods (Wilson et al. 2004). These chemical compounds are mostly used in the hospitals to clean their environment in order to eliminate/reduce the presence of these group of 
organisms' in their environs knowing fully the health implication. This study therefore aims to isolate, identify and investigate the occurrence of airborne fungal spores present in various working environments of eight different hospitals within Lagos State, Nigeria and ascertain the claims of these hospitals on the use of disinfectants to curb this contamination.

\section{MATERIALS AND METHODS}

Sampling area: Eight general hospitals (secondary healthcare) in Lagos state, Nigeria were selected for sampling with the permission from Permanent Secretary, Lagos Health Service Commission (LSHSC/2222/VOL.X/130). Air sampling of various working environment in these hospitals. The area sampled in these hospitals include; the reception, outpatient, male ward, female ward, maternity ward and operating theatre.

Air sampling procedure: Air samples were obtained at each working area by exposing previously fresh prepared Sabouraud dextrose agar (with chloramphenicol) for five minutes. Triplicate of SDA plates were exposed at different angle of constant height of about 1.5 above the floor in each sampled area. This air sampling was carried at the same time, between 11 a.m. - 12 p.m. of each sampled day as this is the peak period for the influx of patients and medical personnel at these sampling sites. The exposed petri plates were transported to the laboratory in sealed zip locked bags. The sampling was conducted every week days (Monday - Friday) for six consecutive weeks (April $9^{\text {th }}-$ May $17^{\text {th }}$ ).

Mycological examination of airborne samples: A total of 90 culture sample plates were exposed at each hospital, incubated at $28^{\circ} \mathrm{C}$ and observed daily. The fungi were subsequently identified by both microscopic and macroscopic observation of each colony isolated. The identities of these fungi were certified by comparing them with confirmed representatives of different species in relevant mycology texts; Ellis et al. (2007).

\section{RESULTS AND DISCUSSION}

Seven species of fungi belonging to three genera were recovered from the study; Aspergillus niger, A. flavus, A. fumigatus, A. species, Candida species, Penicillium citrinum and P. species (Plate 1 - 7). The most abundant fungus encountered in this study is A. niger (fig. 1-6) with P. species being the least isolated fungus from all the investigated hospitals.
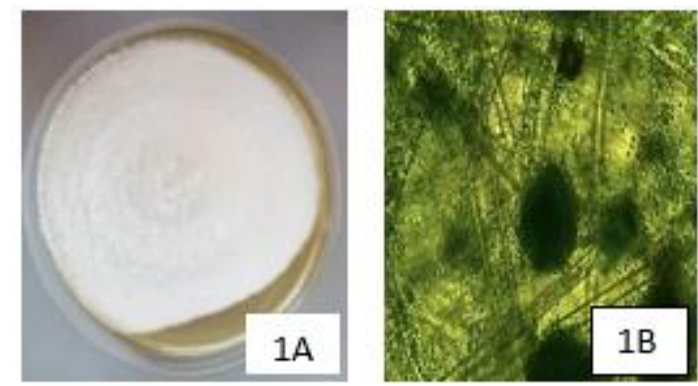

Plate 1a\&b: culture plate and micrograph of Aspergillus species

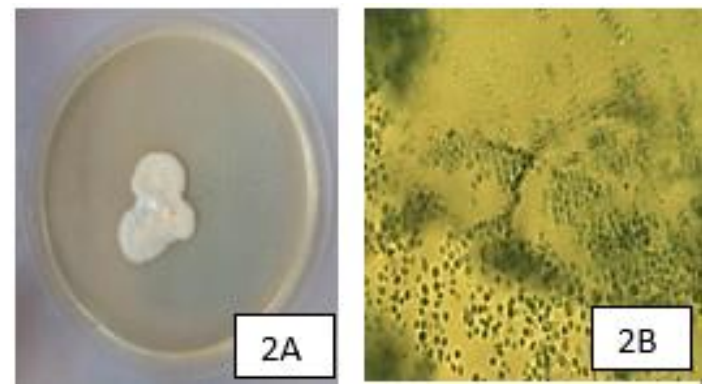

Plate 2a\&b: culture plate and micrograph of Candida species

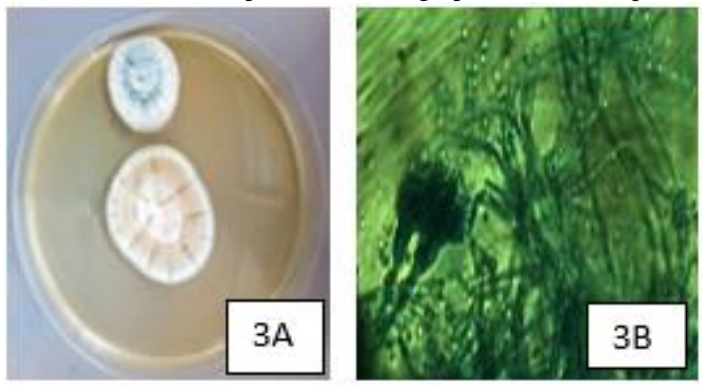

Plate 3a\&b: culture plate and micrograph of Penicillium citrinum
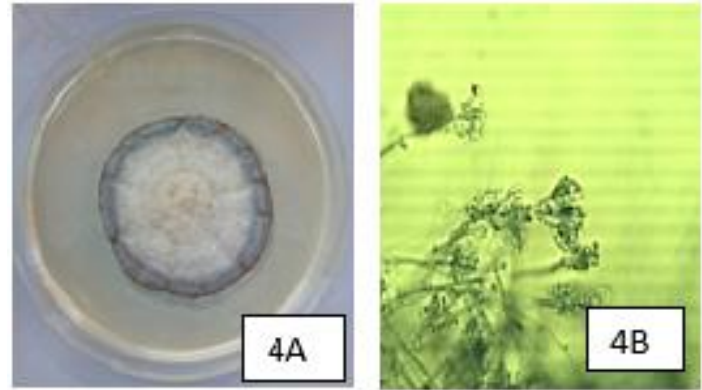

Plate 4a\&b: culture plate and micrograph of Penicillium species
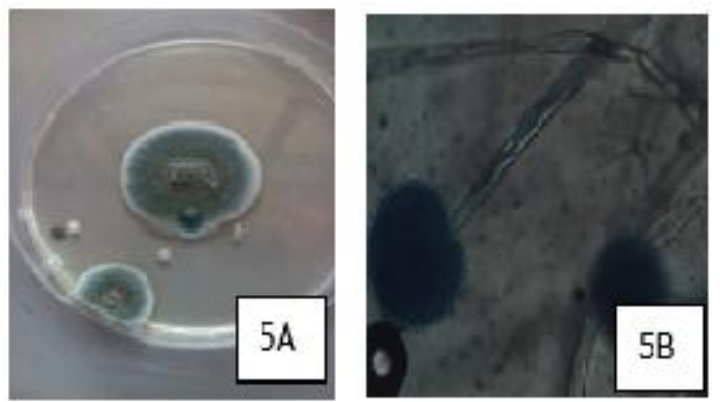

Plate 5a\&b: culture plate and micrograph of Aspergillus fumigatus 

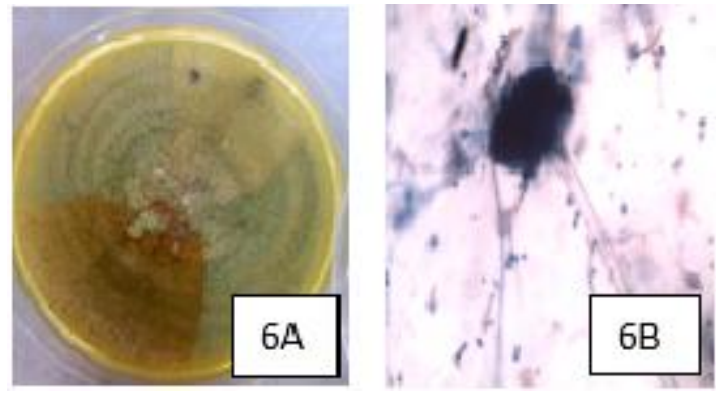

Plate 6a\&b: culture plate and micrograph of Aspergillu flavus

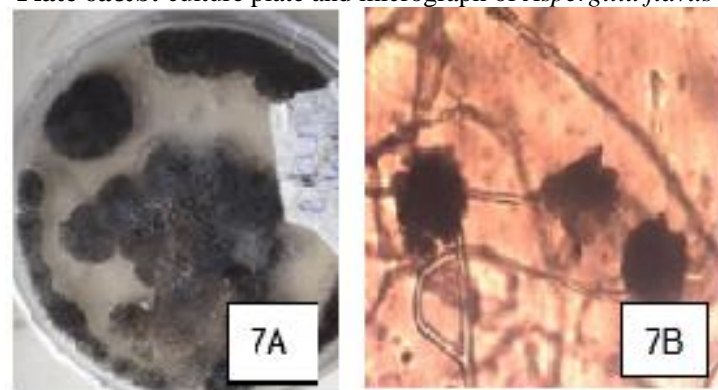

Plate 7a\&b: culture plate and micrograph of Aspergillus niger

Of the six working areas sampled in the eight hospitals, the reception and out-patient areas appeared to record the highest occurrence of encountered fungi (fig. 1 and 2). This trend was also observed in the male and female wards of the investigated hospitals (fig. 3 and4). The reception areas of all the sampled hospitals recorded highest occurrence of $A$. niger, having isolation range between 87 - 90 plates of the total 90 culture plates exposed at each hospital (fig. 1) with an exception to hospital 2. At hospital 2, A. species which was found to be present in the reception of this hospital but absent in all other seven hospital receptions sampled (fig 1). A. niger, was also found to be the most abundant in all the out-patient areas investigated but absent completely in hospital 8 throughout the study period (fig 2). Hospital 8 out-patient area appears to be the only area among that of the eight study hospital with the presence of $A$. species (fig. 2). Same trend was observed with the fungus Candida species, which was only noted at out-patient area of hospital 7 (fig. 2).

The male wards areas of the investigated hospitals were found to be contaminated with all above listed fungal spores except for A. species which was observed to be absent all through the sampled period (fig. 3). Candida species on the other hand, was observed to be the most frequently isolated organism in the eight female wards examined (fig. 4). The fungus Candida was also noted to be the most abundant fungus (fig. 5) in all investigated maternity wards. Though, the occurrence in the maternity wards seem lower compare to that encountered in the sampled female wards. From fig. 6, it was observed that the examined operating theatre rooms had the least fungal contaminant occurrence. Penicillium species for instance, was not recovered from these sampled areas and P. citrinum was only observed in the operating theatre of hospital 8 on just two culture plates of the 90 exposed culture plates.

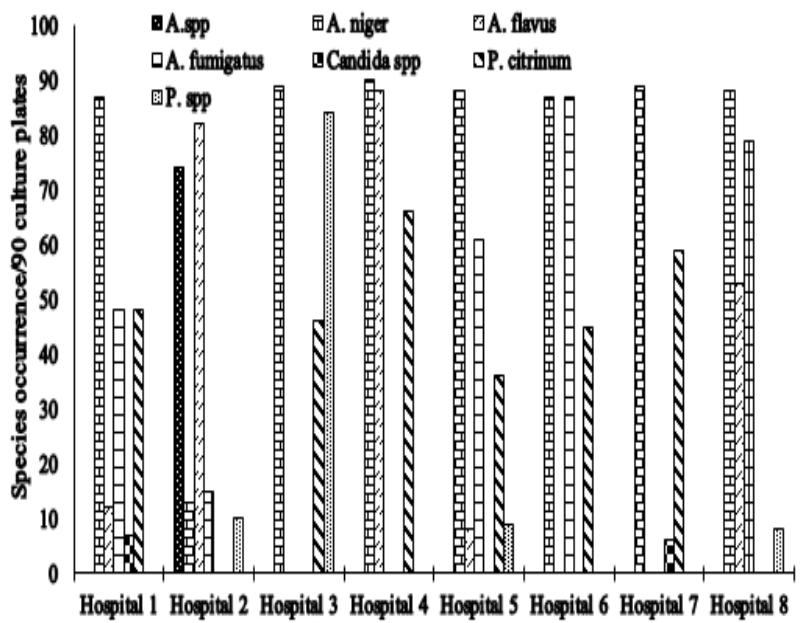

Fig. 1: occurrence of fungi in receptions of all sampled hospitals

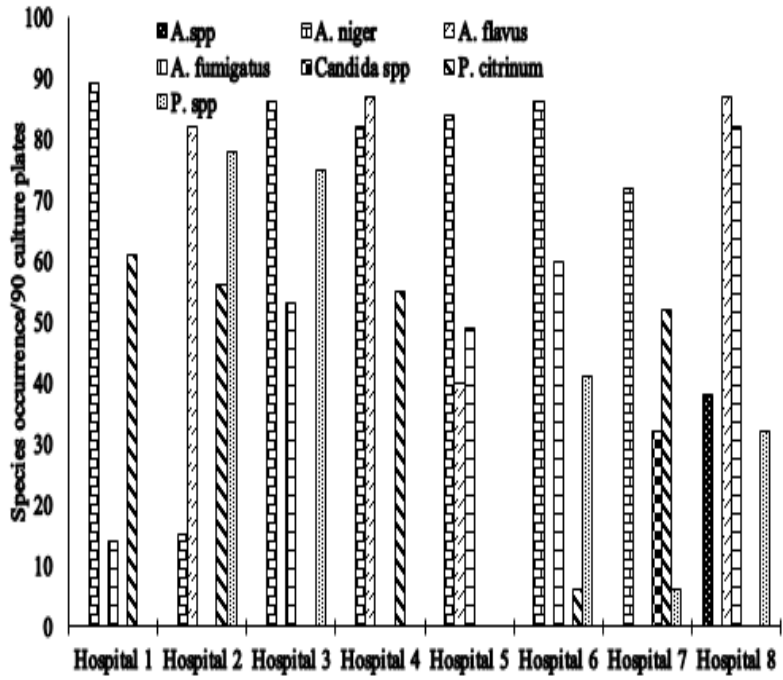

Fig. 2: occurrence of fungi in out-patient wards of all sampled hospitals

It was deduced from the results that various degrees of variety of pathogenic fungi contamination are present in all the eight hospital environments examined in this study despite their claims on the use of disinfectant on daily basis to clean. Hospital, an institution ought to provide patient with treatment through specialized medical personnel now appears to source of many nosocomial fungal infections to the patients. This is due to the encountered load/occurrence of fungal airborne spores in the investigated hospitals. 


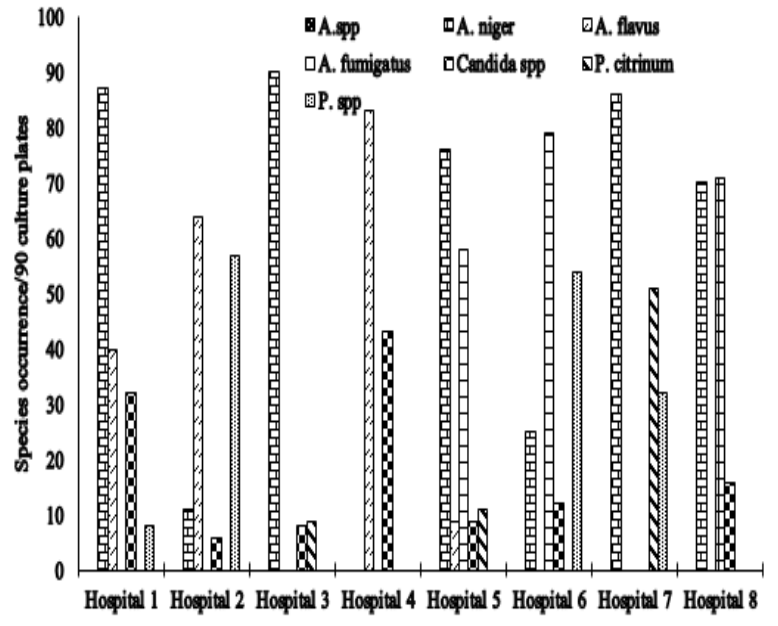

Fig. 3: occurrence of fungi in male wards of all sampled hospitals

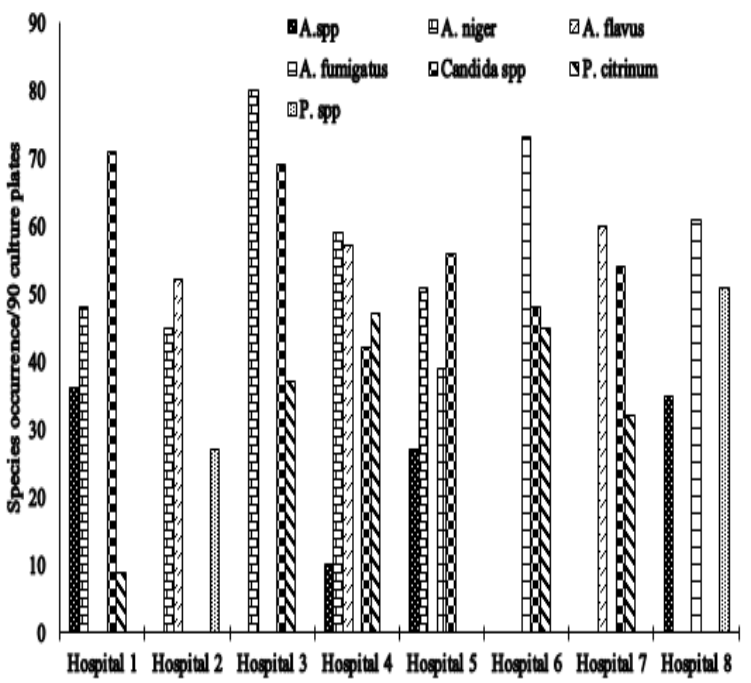

Fig. 4: Occurrence of fungi in female wards of all sampled hospitals

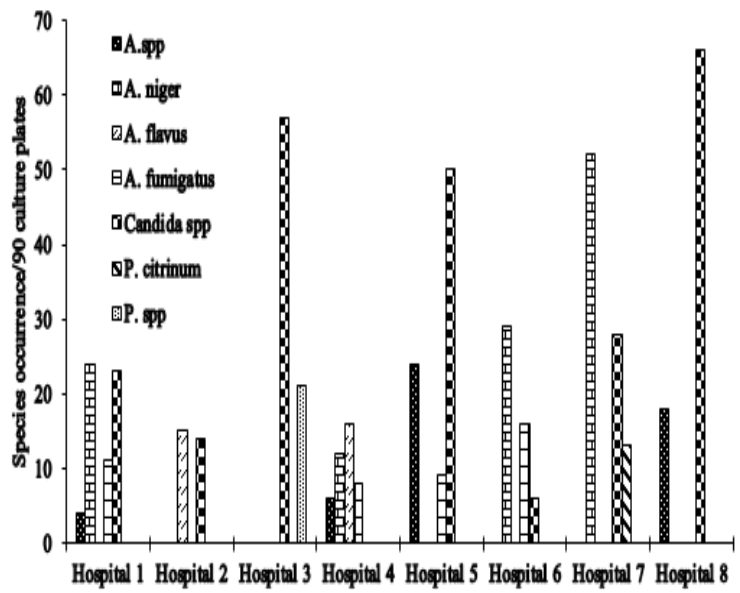

Fig. 5: Occurrence of fungi in maternity wards of all sampled hospitals

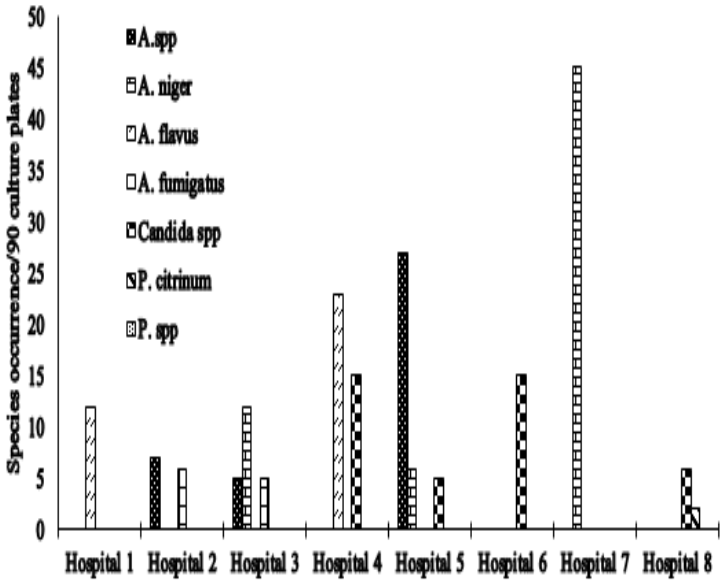

Fig. 6: Occurrence of fungi in operating theatres of all sampled hospitals

The isolated species of fungi from this work are consistent with those reported by streifel et al. (1987); Saadoun et al. (2008); Bifarin et al. (2013); Aglawe et al. (2015); Martinez-Herrera et al. (2016) and Mousavi et al. (2016). Species of Aspergillus recovered in this work were in accordance with the work of Hedayat et al. (2004). These authors stated that species of this genera are ubiquitous mold in homes and hospital environments. Mousavi et al. (1995) reports fungi as one of the important producer of allergens which can caused diseases including localized infection, fatal diseases, allergic responses in human when inhaled their spores/conidia. $A$. fumigatus which is one of the most isolated fungal species in this work, was reported as a life-threatening airborne opportunistic fungal pathogen which is particularly important among immunocompromised patients (Patterson and Strek, 2010). Segal and Romoni, (2009) also pointed inhaling spores of $A$. fumigatus intothe lungs of human may cause a serious hazard and the severity of this depends on the immunological status of the host. Storti et al. (2012) stated that patient own body is usual the reservoir of Candida species and Pfaller and Diekema (2007) reported that over $75 \%$ of women will suffer from Candida albicans infection in their life-time. They also reported Candida species being the fourth most caused of nosocomial infection in patients in the United States. This may justify the reason why occurrence of Candida species was more in the sampled female wards and maternity. Cook et al. (2007) and Bliss et al. (2008) in their work concluded that nosocomial fungal infections may originate from patients themselves into the hospital environment or transmitted to patients through contaminated biomedical devices or even by hospital personnel. According to Storti et al. (2012), they noticed that constant contact with patients in a hospital 
environment facilitates cross-contamination inside the wards (e.g. female wards and maternity). Deorukhkar and Saini (2016), in their study concluded that any microorganism is capable of causing nosocomial infections, but resistance to antimicrobial agents and disinfectants will eventually be established over time by those capable of surviving and persisting in the hospital environment. This explains why despite the use of different disinfectants in the working environments in the sampled hospitals, loads of fungal spore contamination were still recovered. The low level of fungal spore contamination observed in the investigated operating theatres and maternity wards, according to Sessa et al. (2002); Saad, (2003) and Saadoun et al. (2008) may be as the result of factors like number and activities of patients, design of theatre rooms which prevent outdoor air and dust from entering and disinfectant process and methods.

Conclusion: In conclusion, it was gathered from this study that despite the daily cleaning of the investigated hospital working environments with different antiseptic and disinfectants, fungal contaminant was observed throughout the sampled period. We thereby conclude that the hospital environments are continuously monitored in order to identify and eliminate the sources of these fungi. Further study is also recommended to assess the safety of the hospital environments and define new preventive measures and control.

Acknowledgement: We (the authors) appreciate Lagos Health Services Commission for the permission to access the hospitals for this study.

\section{REFERENCES}

Aglawe, V; Mir, MA; Patel, S; Sontakke, H (2015). Isolation of opportunistic pathogenic fungal contamination from hospital environment. Int $J$ Glob Sci Res. 2(3): 213-220.

Bifarin, OO; Feruke-Bello, YM; Komolafe, O (2013). Microbiological examination of air in the neonatal ward of a teaching hospital in Nigeria. Res. Environ. Life Sci. 6(2): 37-40

Bliss, JM; Basavegowda, KP; Watson, WJ; Sheikh, AU; Ryan, RM (2008). Vertical and Horizontal Transmission of Candida albicans in Very Low Birth Weight Infants Using DNA Fingerprinting Techniques. Pediatric Inf Dis J. 27: 231-235

Carris, LM; Little, CR; Stiles, CM (2012). Introduction to Fungi. The Plant Health Instructor. DOI:10.1094/PHI-I-2012-0426-01
Cook, HA; Cimiotti, JP; Della-Latta P, Saiman L, Larson, EL (2007). Antimicriobial resistance patterns of colonizing flora on nurse's hands in the neonatal intensive care unit. Am J Infect Control. 35: 231-236.

Cross, S (1997). Mould spores: the unusual suspects in hay fever. Community Nurse 3: 25-26.

Deorukhkar, SC; Saini, S (2016). Why Candida Species have Emerged as Important Nosocomial Pathogens? Int. J. Curr. Microbiol. App. Sci. 5 (1): 533-545

Ellis D; Davis S; Alexiou H; handke R; Bartley R (2007). Descriptions of medical fungi. Second edition. Nexus Print Solutions, Underdale, South Australia. 198pp.

Hedayati, MT; Mohseni-Bandpi, A; Moradi, SA (2004). Survey on the pathogenic fungi in soil samples of potted plants from Sari Hospitals, Iran. J Hosp Infect. 58(1):59-62.

Hedayati, MT; Pasqualotto, AC; Warn, PA; Bowyer, P; Denning, DW (2007). Aspergillus flavus: human pathogen, allergen and mycotoxin producer. Microbiology 153(6):1677-1692.

Małgorzata, J (2014). Aeromycology: studies of fungi in aeroplankton, Folia Biologica et Oecologica. 10: $18-26$

Martínez-Herrera, EO; Frías-De-León, MG; DuarteEscalante, E, Calderón-Ezquerro, MC, JiménezMartínez, (2016) MC, Acosta-Altamirano G, et al. Fungal diversity and Aspergillus in hospital environments, Ann Agric Environ Med. 23(2): 264-269.

McDonnell G; Russell AD (2001). Antiseptics and Disinfectants: Activity, Action, and Resistance Clin. Microbiol. Rev. 12(1): 147-79

Meers, PD; Ayliffe, GA; Emmerson JA; Leigh DA (1990). Report of the national survey of infection in hospitals. Journal of Hospital Infection. 2: 1-11

Mousavi B; Hedayati MT; Hedayati N; Ilkit M; Syedmousavi S (2016). Aspergillus species in indoor environments and their possible occupational and public health hazards. Curr. Med Mycol. 2(1): 36-42

Patterson, K; Strek, ME (2010). Allergic bronchopulmonary aspergillosis. Proc Am Thorac Soc. 7(3): 237-44. 
Pfaller, M; Diekema, D (2007). Epidemiology of invasive candidiasis: A Persistent public health problem. Clin. Microbiol. Rev. 20: 133 - 63

Saad, S G (2003). Integrated environmental management for hospitals. Indoor Built Environ 12: $93-98$

Saadoun I; Al Tayyar IA; Elnasser Z (2008). Concentrations of Airborne Fungal Contamination in the Medical Surgery Operation Theaters (OT) of Different Hospitals in Northern Jordan. Jordan J. Biol. Sci. 1(4): 81 - 184

Segal, BH; Romani, LR (2009). Invasive aspergillosis in chronic granulomatous disease. Med. Mycol. 47: S282-S290

Sessa, R; Di, PM; Schiavoni, G; Santino, I; Altieri, A; Pinelli, S; Del, PM (2002). Microbiological indoor air quality in healthy buildings. New Microbiology. 2002; 25: 51-56

Southworth, D (1974). Introduction to the biology of airborne fungal spores. Annals of Allergy 32: 1-22.
Storti, LR; Pasquale, G; Scomparim, R; Galastri, AL; Alterthum, F; Gambale, W; Paula, CR (2012). Candida spp. isolated from inpatients, the environment, and health practitioners in the Pediatric Unit at the Universitary Hospital of the Jundiaí Medical College, State of São Paulo, Brazil. Revista da Sociedade Brasileira de Medicina Tropical. 45(2): 225-231

Streifel, AJ; Stevens, PP; Rhame, FS (1987). InHospital Source of Airborne Penicillium Species Spores. J. Clin. Microbiol. 25(1): 1-4

Tainter, FH; Baker, FA (1996). Principles of forest pathology. New York, USA, John Wiley \& Sons. 832 pp.

Wilson APR; Gibbons C; Raeves BC; Hogson B; Lius M; Plummer D (2004). Surgical wound infections as a performance abdicator agreement of common disinfection of wound infections in patients. Am J Infect Control. 329: 720-925 